\title{
Artefato Metodológico de Autoria Aplicado aos Mundos Virtuais para Educação
}

\author{
Leander C. de Oliveira ${ }^{1}$, Danúbia B. Espíndola ${ }^{1}$, Regina Barwaldt ${ }^{1}$, Marília A. \\ Amaral $^{2}$, Silvia S. C. Botelho ${ }^{1}$ \\ ${ }^{1}$ Centro de Ciências Computacionais (C3) - \\ Universidade Federal do Rio Grande (FURG) - \\ Rio Grande - RS - Brasil \\ ${ }^{2}$ Departamento Acadêmico de Informática (DAINF) - \\ Universidade Tecnológica Federal do Paraná (UTFPR) - \\ Curitiba - PR - Brasil. \\ \{leanderdeoliveira, danubiafurg\}@gmail.com, reginabarwaldt@furg.br, \\ \{silviacb.botelho, marilia.utfpr\} @gmail.com
}

\begin{abstract}
By continuous search for the convergence between technology and education, there are some studies involving virtual worlds in this context. By observing low applicability and dissemination of this technology, it is important to have a debate about the authorship of these environments. In the research described here, we intended to develop a methodological artifact that aims to guide and motivate the development and consequent use of virtual worlds in educational contexts.
\end{abstract}

Resumo. Por meio da busca contínua pela convergência entre tecnologia e educação, nota-se a existência de alguns estudos envolvendo Mundos Virtuais neste contexto. Ao observarmos pouca aplicabilidade e disseminação desta tecnologia, torna-se importante criar um debate sobre a autoria destes ambientes. Pela pesquisa aqui descrita, pretendeu-se desenvolver um artefato metodológico que objetiva guiar e motivar o desenvolvimento, e a consequente utilização de Mundos Virtuais em contextos educativos.

\section{Introdução}

Embora exista uma crescente disseminação dos ambientes virtuais de aprendizagem (AVAs) (Belmonte e Grossi, 2010; Gabardo et. al., 2010), as metodologias e ferramentas de autoria descritas na literatura ainda não abrangem de forma concisa os Mundos Virtuais (MV) para fins educativos. Estes Mundos Virtuais Educacionais, também denominados como metaversos, possibilitam a imersão do aprendiz através de visualização tridimensional (3D) e representação dos utilizadores por meio de avatares digitais em ambientes educacionais, laboratórios, congressos, jogos e diferentes contextos ainda a serem explorados (Lorenzo et. al., 2012; Viegas, 2012; Nascimento e Spilker, 2012). Além deste tipo de ambiente permitir aos atores interações de aprendizagem cotidianas, ele pode ter características meta-realísticas. Esta distinção permite o desenvolvimento de realidades virtuais que podem ser ampliadas (ou "melhoradas"), permitindo o desenvolvimento de Mundos Virtuais que possibilitam diferenciar o processo educacional com rompimentos no contexto de realidade 


\section{CBIE-LACLO 2015}

Anais dos Workshops do IV Congresso Brasileiro de Informática na Educação (CBIE 2015)

(Schlemmer e Marson, 2013; Nascimento e Spilker, 2012). O termo metaverso (meta universo) deriva justamente desta característica.

A autoria de conteúdos para Mundos Virtuais Educacionais, por meio de ferramentas de desenvolvimento, é muitas vezes, um processo complexo (Ávila et. al., 2014; Khot e Choppella, 2011). A possibilidade de desenvolvimento orientado pelo professor demanda horas para o desenvolvimento de conteúdos 3D interativos e culminam, frequentemente, na desistência de propor conteúdos mais elaborados (Ávila et. al., 2014; Khot e Choppella, 2011). Considerando estes fatos e a necessidade de trazer a autoria de ambientes complexos para as mãos do professor, é preciso estabelecer um debate acerca deste processo de elaboração. Quais são as etapas envolvidas no desenvolvimento de conteúdos para Mundos Virtuais Educacionais e como considerálas para que o processo de autoria seja simples e motivador aos autores/docentes?

Aliada à definição de uma abordagem metodológica, foi objetivo deste estudo elencar um conjunto de aplicações e ferramentas que permitam ao autor/docente expor seus conceitos e conteúdos. A preocupação com este processo é que os conteúdos e materiais sejam apresentados de maneira síncrona, considerando estágios e etapas para o aprendizado.

No intuito de investigar os temas da pesquisa, foi desenvolvida uma revisão sistemática apresentada na seção 2. Esta revisão buscou quantificar e apontar a necessidade dos debates propostos por esta pesquisa. $\mathrm{O}$ apoio de uma abordagem de pesquisa que centralize o usuário no processo de desenvolvimento se torna importante a partir do momento em que as soluções que abordam os Mundos Virtuais Educacionais são poucas vezes observadas em contextos práticos nas instituições de ensino. A seção 3 apresenta o artefato metodológico desenvolvido como proposta à problemática apresentada.

Conforme diretrizes do design de interação (Preece et. al., 2005), a participação de agentes/atores torna-se importante na criação do artefato proposto, pois assim orienta-se o desenvolvimento conforme necessidades e expectativas do público ao qual este se destina. Para que pudesse ser proposto um processo de apropriação tecnológica de forma consistente, aconteceram interações com alguns potenciais multiutilizadores destas tecnologias (autores) na busca pelas etapas, ferramentas e validação do artefato desenvolvido. Esta socialização está apresentada na seção 4.

\section{Revisão Sistemática}

Como forma de compreender o objeto de estudo debatido nesta pesquisa, foi desenvolvida uma revisão sistemática de literatura. Uma revisão sistemática de literatura pode ser utilizada para identificar, interpretar e avaliar pesquisas disponíveis e que estejam em coerência com o tema estudado (Kitchenham, 2004). Foram definidos seis repositórios de artigos, onde foram utilizadas seis palavras-chave como forma de obter artigos para a análise posterior.

Os repositórios e seus links para acesso são: $\mathrm{RBIE}^{1}, \mathrm{Renote}^{2}, \mathrm{SBIE}^{3}, \mathrm{WIE}^{4}$, $C \& E^{5}$ e IEEE ${ }^{6}$. As palavras-chave e suas traduções foram: Ambiente virtual (Virtual

\footnotetext{
${ }^{1}$ Revista Brasileira de Informática na Educação 〈http://www.br-ie.org/pub/index.php/rbie >
} 
Enviroments), Ambientes imersivos (Immersive Enviroments), Autoria (Authorship), Avatar (Avatar), Metaversos (Metaverses) e Mundos Virtuais (Virtual Worlds).

Após as definições iniciais da revisão, das triagens e das classificações dos artigos, obteve-se o grafo da Figura 1 que demonstra a classificação dos artigos conforme diferentes categorias.

Figura 1. Grafos de classificação dos artigos

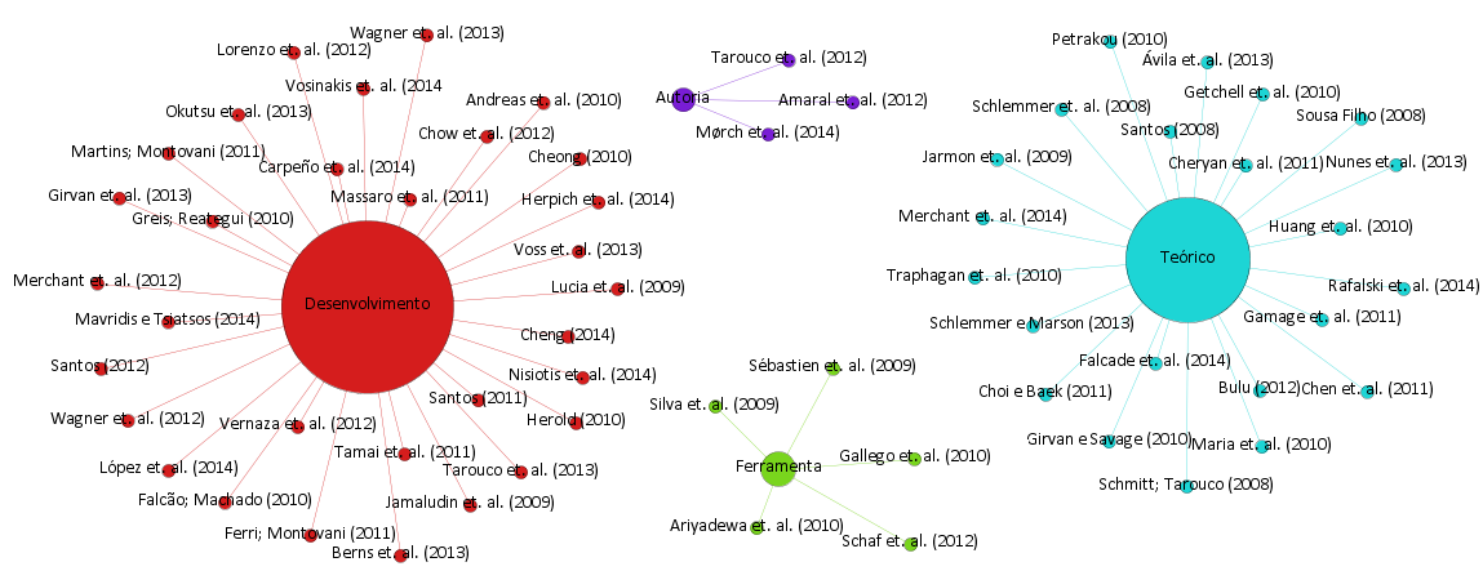

Fonte: Autor

Conforme observado, foram selecionados 61 artigos destes repositórios. Apenas três $(4,91 \%)$ tiveram alguma relação com a autoria em Mundos Virtuais. Dos demais, 31 foram classificados como "Desenvolvimento" (50,81\%), trabalhos cujo principal objetivo foi descrever o desenvolvimento de Mundos Virtuais, Objetos Virtuais de Aprendizagem (OVAs), estudos de caso. Vinte e dois como "Teóricos" (36,06\%), pesquisas que se referem a características, efeitos, possibilidades dos ambientes. Cinco como "Ferramenta" $(8,19 \%)$, trabalhos com descrição de experimentos e ferramentas com caráter genérico, aplicáveis à outros estudos.

Ainda que os trabalhos tratem de temas relacionados à autoria, não tiveram como foco uma sistematização ou organização de etapas para desenvolvimento dos ambientes. Tarouco et. al. (2012) descreve a possibilidade dos alunos em interagir e criar objetos e elementos e, desta forma, compreender os conceitos envolvidos. Isso faz com que o estudante também possa ser compreendido como autor no processo de ensino e aprendizagem. $O$ estudo também descreve ferramentas facilitadoras para 0 desenvolvimento de elementos aplicados à Mundos Virtuais, descritas como ferramentas para a autoria. Outro artigo (Amaral et. al., 2012), que está relacionado ao anterior, também discute a autoria e aponta a necessidade de selecionar ferramentas facilitadoras aos autores/multiusuários dos ambientes. São descritas ferramentas, mas sem maiores detalhamentos. Já o trabalho de Mørch et. al. (2014) descreve um estudo qualitativo que apontou um conjunto de etapas de design relacionadas à utilização destes ambientes. São citados os seguintes tipos de dependências para o planejamento de conteúdos em

\footnotetext{
${ }^{2}$ Revista Novas Tecnologias na Educação <http://seer.ufrgs.br/renote/>

${ }^{3}$ Simpósio Brasileiro de Informática na Educação 〈http://www.br-ie.org/pub/index.php/sbie >

${ }^{4}$ Workshop de Informática na Escola 〈http://www.br-ie.org/pub/index.php/wie/issue/archive >

${ }^{5}$ Revista Computers \& Education <http://www.sciencedirect.com/science/journal/03601315>

${ }^{6}$ Repositório IEEE <http://ieeexplore.ieee.org/Xplore/home.jsp>
} 
MV: (i) Aplicação de interação social no contexto tratado; (ii) Acompanhamento e suporte à aprendizagem dos alunos sobre a nova tecnologia; (iii) (a) Planejamento da estrutura do curso e das aulas; e (b) Tratamento acerca dos procedimentos que deverão ocorrer no Mundo Virtual; (iv) Tratamento das transições entre atividades.

A análise quanti-qualitativa possibilitada por esta revisão sistemática, proporcionou entendimento acerca da aplicabilidade e importância de um estudo que proponha processos para a autoria de Mundos Virtuais em contextos educacionais.

\section{Artefato Metodológico}

Conforme os objetivos apresentados, as etapas de autoria foram compreendidas por meio de análise bibliográfica (Ávila et. al., 2013; Tarouco et. al., 2013; Wagner et. al., 2012; Amaral et. al., 2012; Tarouco et. al., 2012; Ferri e Mantovani, 2011; Ariyadewa et. al., 2010) e estão representadas graficamente pela Figura 2.

Figura 2. O Artefato Metodológico
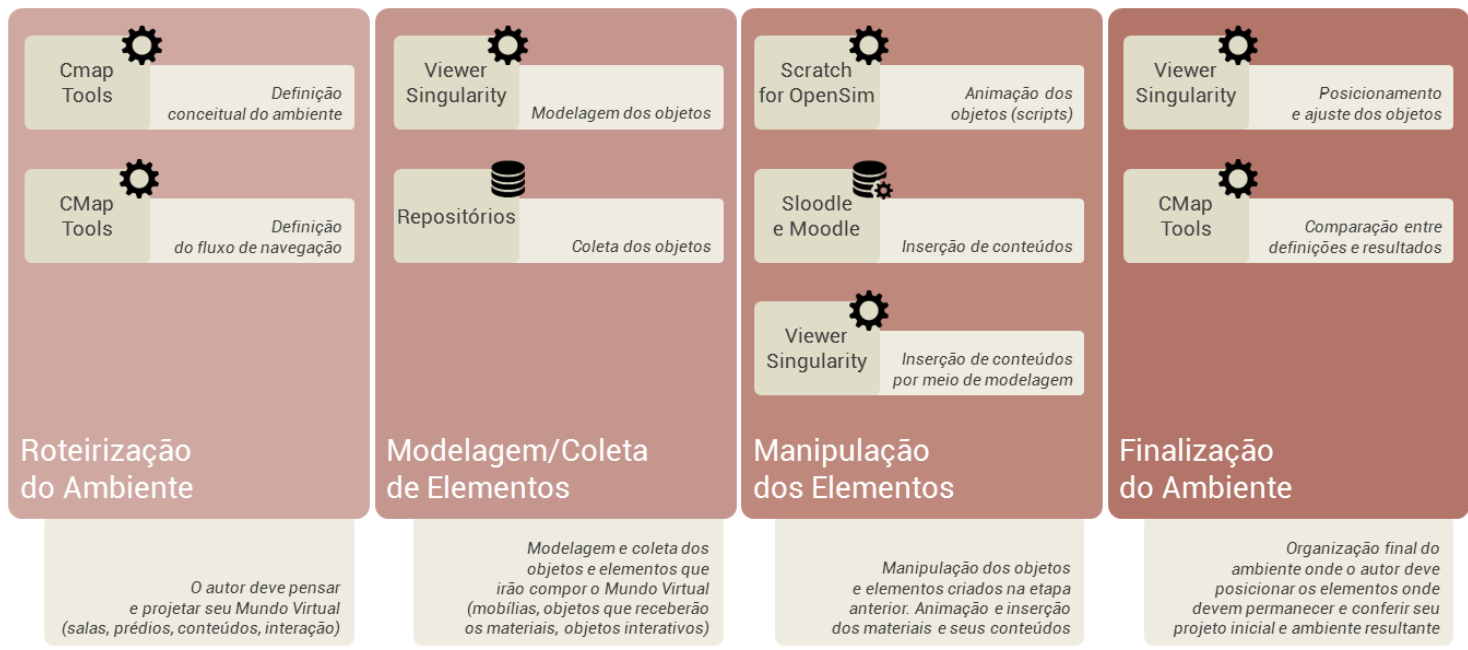

Fonte: Autor

Os desenvolvimentos apresentados nestas pesquisas permitiram compreender as fases para o desenvolvimento. Além das etapas, os estudos apontaram ferramentas que podem ser utilizadas no processo de desenvolvimento (Ávila et. al., 2013; Nunes et. al., 2013; Voss et. al., 2013; Tarouco et. al., 2012; Amaral et. al., 2012; Mota et. al., 2012), conforme ilustrado na figura. Dois artigos foram publicados pelos autores e estão relacionados a esta etapa do trabalho. Em um dos artigos (Oliveira et. al., 2014) é apresenta a proposta do artefato metodológico com a descrição de alguns testes ferramentais e de desenvolvimento. O outro artigo (Oliveira et. al., 2014b) relaciona a autoria de Mundos Virtuais com o Design Participativo e apresenta uma análise acerca das ferramentas de autoria, suas aplicações e possibilidades.

\subsection{Roteirização do Ambiente}

Existem dois momentos nessa etapa do desenvolvimento, conforme mostra o diagrama apresentado na Figura 2. O primeiro diz respeito às definições conceituais do ambiente e o segundo ao fluxo de navegação que irá definir as interações neste ambiente. Alguns autores, quando descrevem a implementação de seus Mundos 


\section{CBIE-LACLO 2015}

Anais dos Workshops do IV Congresso Brasileiro de Informática na Educação (CBIE 2015)

Virtuais, apresentam maior destaque para esta etapa. Tarouco et. al. (2013), Tarouco et. al. (2012) e Amaral et. al. (2012) apresentaram, no início de suas descrições sobre a implementação do ambiente, o roteiro em desenvolvimento na pesquisa e que mostra a estrutura pedagógica do ambiente. Ferri e Mantovani (2011), por sua vez, descrevem a necessidade de modelar seus ambientes por meio de um Planejamento TécnicoDidático-Pedagógico e outro Urbano e Arquitetônico. Dentre as diretrizes apontadas por Mørch et. al. (2014), já citadas anteriormente, o terceiro ponto é o que possui maior relação com esta etapa: (a) planejamento da estrutura do curso e das aulas; (b) tratamento acerca dos procedimentos que deverão ocorrer no Mundo Virtual.

Uma importante questão a ser considerada no desenvolvimento de conteúdos para Mundos Virtuais é a organização da apresentação do conteúdo de forma tridimensional (Schlemmer e Backes, 2008). Com o planejamento inicial do curso, chegou-se ao segundo momento desta etapa. Este momento esteve em convergência ao quarto ponto definido por Mørch et. al. (2014), que é o tratamento das transições entre as atividades. A organização dos fluxos de navegação do aprendiz no ambiente pode ser um fator importante no processo de autoria e, em consequência, na efetividade da apresentação dos conteúdos em questão. Com este propósito, torna-se necessário contextualizar os materiais na maneira como serão tratados e apresentados no ambiente, trazendo um significado para eles no processo educacional do ambiente.

Dessa forma, esta etapa do desenvolvimento, em resumo, segue um fluxo que inicia com o autor pensando o seu ambiente de maneira ampla e abrangente, respondendo a questões como: (i) quais os conteúdos, conceitos e materiais serão abordados; (ii) qual será a abordagem de linearidade do ambiente e, consequentemente, quais caminhos o avatar poderá seguir; (iii) em quais espaços os avatares poderão interagir; (iv) como essa interação pode ocorrer; (v) quais objetos estarão presentes.

\subsection{Modelagem/Coleta dos Elementos}

Com o planejamento e roteirização do ambiente feitos, esta etapa trata da criação ou pesquisa dos objetos que são necessários para o desenvolvimento do Mundo Virtual. Como pôde ser observado no diagrama (Figura 2) existem duas possibilidades para este momento do desenvolvimento. Algumas pesquisas sobre Mundos Virtuais explicam em suas implementações como ocorre a coleta dos elementos que compõem o ambiente planejado. Esta etapa pode ser reconhecida nas implementações dos trabalhos de Ávila et. al. (2013) e Amaral et. al. (2012). Estes autores descrevem diferentes ferramentas para a modelagem de elementos, dentre elas podem ser destacadas SketchUp, Blender ou ainda os próprios viewers usados para acessar os Mundos Virtuais. A outra possibilidade além da modelagem dos objetos é a utilização de repositórios online de objetos e elementos. Existem variados repositórios de objetos e elementos prontos que podem ser inseridos de maneira simples em um Mundo Virtual, o que descomplica o processo de criação (Ávila et. al., 2013; Amaral et. al., 2012).

\subsection{Manipulação dos Elementos}

Com os objetos modelados, esta etapa tratou da manipulação destes objetos, ou seja, a definição e implementação das animações e a inserção dos conteúdos multimídia que farão parte do Mundo Virtual. É nesta etapa também que os elementos coletados em repositórios foram adaptados e manipulados para atenderem ao que foi predefinido para 


\section{CBIE-LACLO 2015}

Anais dos Workshops do IV Congresso Brasileiro de Informática na Educação (CBIE 2015)

o ambiente em desenvolvimento. Um exemplo desta adaptação foi descrita na implementação da pesquisa de Tarouco et. al. (2013) com um prédio sendo coletado em um repositório online e editado usando o viewer para atender as necessidades do ambiente descrito no trabalho. A estrutura do prédio foi manipulada e novas salas foram adicionadas à construção original, assim atendendo às predefinições do projeto.

Outro importante ponto tratado neste momento do desenvolvimento foi a criação dos scripts que permitiram aos objetos tornarem-se interativos no ambiente. Ávila et. al. (2013) descreve em seu trabalho algumas possibilidades como a criação de scripts utilizando linguagens próprias de cada tecnologia, como a OSSL (OpenSim Scripting Language) para o OpenSimulator, por exemplo. Também são descritas ferramentas que facilitam a geração destes scripts por meio de programação visual, como o Scratch for OpenSim , o ScripTastic e o FS2LSL (Flash Scratch to LSL) (Ávila et. al., 2013).

Conforme as decisões iniciais do autor, a questão da linearidade do ambiente pode ser tratada neste momento do desenvolvimento. Quando existir o objetivo em desenvolver uma roteirização com uma estruturação mais rígida no ambiente, existem diferentes possibilidades e caminhos a serem seguidos. Uma delas é uma abordagem que não limita o avatar em criar seus próprios caminhos no ambiente, mas fornece a ele possibilidades em escolher entre um roteiro predefinido pelo autor ou seu próprio desejo de exploração. Isso pode ser elaborado com a inserção de indicações sobre a sequência dos espaços onde os avatares devem circular utilizando indicações, numerações, setas ou outros elementos que podem guiar a navegação. Outra possibilidade, que permite bloquear espaços e compor uma hierarquia no caminho do avatar, é a utilização de salas com portas bloqueadas que só podem ser abertas com a utilização de uma senha. Esta abordagem pode ser tratada já como forma avaliativa pelo ambiente e gerar feedbacks acerca do aprendizado construído pelos avatares por meio de seus caminhos e interações.

\subsection{Finalização do Ambiente}

Com as etapas anteriores finalizadas, o último momento do desenvolvimento é quando são organizados nos espaços do ambiente os elementos coletados e desenvolvidos. Desta forma os objetos e elementos são distribuídos no ambiente, conforme previsto, para que o ambiente possa ter seu acesso liberado. É interessante que após a finalização do desenvolvimento sejam revisitados os espaços e desenvolvidos testes em todos os elementos observando a conformidade com o que estava previsto pela etapa inicial para que o ambiente não apresente problemas quando for utilizado.

\section{Socialização do Artefato Metodológico}

Cinco docentes foram convidados a participar dos testes do artefato metodológico. Para a realização do teste, um ambiente experimental foi implementado permitindo aos utilizadores empregarem as abordagens debatidas na pesquisa. Todos os participantes são docentes de ensino superior em áreas distintas: um de tecnologia e sociedade, um de ensino de física, um de engenharia da computação e dois de informática na educação.

Os testes foram desenvolvidos com todos os softwares instalados e executados em uma máquina local. As ferramentas foram instaladas e configuradas para o uso, desta 
forma os utilizadores não precisaram manipular nada relacionado a questões de instalação, apenas utilizaram as ferramentas indicadas. O notebook utilizado nos testes possui processador Intel Core i7, interface gráfica Intel ${ }^{\circledR}$ HD Graphics 3000, memória de 4GB e armazenamento em SSD.

Para a realização dos testes, os utilizadores, individualmente e com acompanhamento do pesquisador, foram convidados a desenvolver uma atividade de autoria guiada por um documento que apresentava o artefato metodológico. Este documento apresentou uma atividade de autoria de um Mundo Virtual por meio da abordagem discutida na pesquisa, com atividades relacionadas às etapas descritas. Essa atividade teve como tema de delimitação o ensino básico de informática, mais precisamente, conhecimentos básicos de hardware. Para o desenvolvimento da atividade, um Mundo Virtual foi configurado previamente em uma ilha experimental e uma construção foi disponibilizada para a utilização de cada um dos participantes.

\subsection{Resultados dos Testes}

O tempo de duração dos testes para cada utilizador foi variável, entre 2 horas e 2 horas e meia. Os tempos conforme cada utilizador foram: Utilizador 1 (2:30), Utilizador 2 (2:20), Utilizador 3 (2:30), Utilizador 4 (2:10) e Utilizador 5 (2:00).

Com as observações e comentários dos participantes, notou-se que alguns tiveram algumas dificuldades iniciais para compreender as ferramentas, interfaces e os termos utilizados. A curva de aprendizagem, nesse caso, influenciou na duração do teste e fez com que os utilizadores fizessem questionamentos ao pesquisador. Esses questionamentos foram, em suma, sobre fatores relacionados à interface das ferramentas, como botões e comandos específicos para os softwares. Após a finalização dos testes (todos os participantes finalizaram as atividades) foram respondidos questionários com o intuito de compreender as percepções dos participantes dos testes acerca da utilização do artefato metodológico, além das considerações acerca de modificações e melhorias no artefato como um todo. Do total de 19 questões, as principais são as observadas na Figura 3.

Figura 3. Questões 10, 11 e 13

\begin{tabular}{|c|c|c|}
\hline 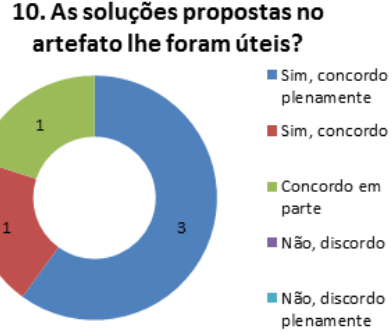 & 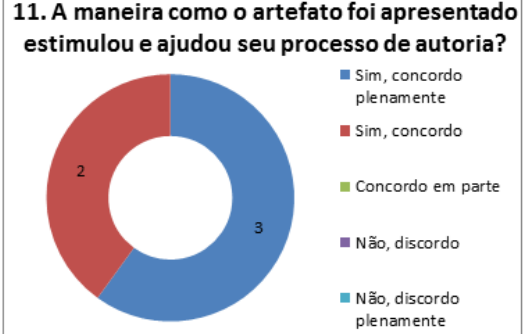 & $\begin{array}{l}\text { 13. Como você classifica a } \\
\text { motivação que sentiu ao utilizar o } \\
\text { artefato? } \\
\begin{aligned} & =\text { Muito alta } \\
& =\text { M Alta } \\
& =\text { Intermediária } \\
& =\text { Maixa } \\
& =\text { Muito baixa }\end{aligned}\end{array}$ \\
\hline
\end{tabular}

Fonte: Autor

A questão 10 pretendia compreender o quanto as soluções foram aceitas pelos participantes. Essas soluções versam sobre a indicação das ferramentas, suas utilizações, as maneiras de organizar as questões de linearidade no ambiente, dentre outras. Pôde ser confirmada uma boa aceitação dos utilizadores referente a este questionamento. A resposta em "Concordo em parte" pode ser compreendida por uma necessidade de testes mais duradouros, para que exista maior tempo de compreensão das ferramentas, 


\section{CBIE-LACLO 2015}

Anais dos Workshops do IV Congresso Brasileiro de Informática na Educação (CBIE 2015)

tecnologias e processos de autoria, o que melhoraria a apropriação das ferramentas por parte dos participantes.

Sobre a maneira como o artefato foi apresentado ter sido estimulante, ajudando o processo de autoria (questão 11), as respostas demonstraram uma boa aceitação, uma vez que foi possibilitado aos utilizadores o desenvolvimento de Mundos Virtuais em um pequeno espaço de tempo, permitindo aos utilizadores a autoria de seus ambientes. A questão 13 pediu aos participantes para classificarem a motivação que sentiram ao utilizar o artefato. Quatro participantes ( $80 \%$ do total) responderam que sentiram motivação alta e um participante, motivação muito alta.

Com as respostas obtidas pode-se concluir que o artefato foi um motivador no processo de autoria/desenvolvimento. Pôde ser percebido durante os testes que os participantes demonstraram interesse pelos Mundos Virtuais. Também foi notada uma importante curiosidade sobre as ferramentas e as aplicações deste tipo de ambiente em contextos educacionais.

\section{Considerações Finais}

A compreensão do estado da arte acerca dos Mundos Virtuais com foco educacional demonstrou a existência de possibilidades inexploradas e desafios complexos para um efetivo uso deste tipo de tecnologia. A partir do momento em que docentes podem se tornar autores de seus ambientes, a motivação e as possibilidades educacionais podem ser potencializadas por meio de fatores como a construção coletiva de conhecimento e o estímulo ao aprendiz por meio da representação e recriação de situações que envolvem sua vivência cotidiana no processo educacional. Com esta motivação em mente, a proposta desta pesquisa buscou alcançar uma maneira de motivar a autoria de Mundos Virtuais em contextos educativos.

Embora as possibilidades de utilização de ambientes desta natureza sejam vastas, conforme demonstra a literatura, ainda existem barreiras que impedem uma utilização plena. O processo de autoria pode ser um dos principais empecilhos na apropriação desta tecnologia por parte dos professores, resultando em pouca disseminação destes ambientes para fins educacionais, conforme debatido por Ávila et. al. (2014). As investigações iniciais, que objetivaram o reconhecimento das etapas genéricas pelas quais passa o processo de autoria, à procura de abordagens de autoria que relacionem os Mundos Virtuais e seus autores, a coleta de informações sobre o ferramental necessário e a posterior prototipação de um artefato metodológico apontaram para estes fins.

Conforme a problemática exposta, foi observado que o artefato de autoria apresentado nesta pesquisa propõe uma maneira clara de ajudar, guiar e facilitar a autoria de conteúdos didáticos para os ambientes virtuais, conforme comprovou-se pelos resultados da socialização do artefato metodológico. Isso faz com que este artefato de autoria possa ser utilizado por multiusuários, grupos de pesquisa, dentre outros interessados, para a autoria de seus conteúdos e elaboração de seus ambientes. A necessidade de um processo de formação ampliado foi um destaque durante os testes, $\mathrm{o}$ que denota o quanto as tecnologias tratadas podem ser complexas e exigem uma preparação e dedicação maiores por parte dos autores. Ainda assim, os participantes dos testes puderam desenvolver os ambientes propostos e responderam às questões de maneira a concluir que o artefato foi efetivo em seu objetivo. Destacado durante os 


\section{CBIE-LACLO 2015}

Anais dos Workshops do IV Congresso Brasileiro de Informática na Educação (CBIE 2015)

testes, por meio de comentários verbais dos participantes, as ferramentas envolvidas no processo de autoria exigem um período de tempo para compreensão e adaptação. Notouse que o início das atividades gerou dúvidas nos participantes, todas questionadas verbalmente. A grande maioria estava relacionada a questões de interface, adaptação de linguagens e termos dos softwares e a quantidade de ferramentas envolvidas.

Com estas considerações, podem ser apontados dois possíveis caminhos para futuros desenvolvimentos relacionados. O primeiro diz respeito a estudos que alavancam as melhorias de software, integrando ferramentas, adequando interfaces, dentre outras possibilidades relacionadas. Uma segunda possibilidade envolveria a formação docente para a utilização das ferramentas disponíveis, o que acaba reiterando a anteriormente destacada necessidade de uma formação ampliada e continuada.

Finalmente, pode ser afirmado que a efetividade e popularização de recursos tecnológicos em contextos educacionais devem ser uma busca contínua, principalmente quando estão envolvidos conceitos abstratos que podem ser beneficiados por modelos visuais para uma melhor compreensão. Ao passo da crescente evolução da convergência entre tecnologia e educação, tornam-se cada vez mais reais as possibilidades de envolver indivíduos e grupos no processo educacional, por meio de situações que tornam este processo cada vez mais abrangente, acessível, efetivo e agradável.

\section{Referências}

Amaral, É.; Avila, B. G.; Tarouco, L.. (2012). Aspectos teóricos e práticos da implantação de um laboratório virtual no OpenSim. XXIII Simpósio Brasileiro de Informática na Educação, Rio de Janeiro, Brasil.

Ariyadewa, P. D.; Wathsala, W.V.; Pradeepan, V.; Perera, R.P.D.D.T.; Atukorale, D.A.S.. (2010). Virtual Learning Model for Metaverses. 11th International Conference on Advances in ICT for Emerging Regions (ICTer), Colombo, Sri Lanka.

Ávila, B.; Amaral, É.; Tarouco, L.. (2013). Implementação de Laboratórios Virtuais no metaverso OpenSim. Revista Novas Tecnologias na Educação (RENOTE), V.11 No1.

Ávila, B.; Tarouco, L.; Passerino, L.; Guterer, P.. (2014). Autoria nos Mundos Virtuais: um novo desafio ao docente. Revista Novas Tecnologias na Educação (RENOTE). V. 12. $\mathrm{N}^{\mathrm{o}} 2$. Acesso em: 28 mar. 2015.

Belmonte, V.; Grossi, M.. (2010). Ambientes Virtuais de Aprendizagem: Um Panorama da Produção Nacional. $16^{\circ}$ Congresso Internacional ABED de Educação a Distância.

Ferri, J.; Mantovani, A.. (2011). Construção Do Espaço Digital Virtual Em Três Dimensões Do Unilasalle No Metaverso Second Life. Revista Novas Tecnologias na Educação (RENOTE), V. $9 \mathrm{~N}^{\circ} 1$.

Gabardo, P.; Quevedo, S.; Ulbricht, V.. (2010). Estudo Comparativo das Plataformas de Ensino-Aprendizagem. Encontros Bibli: Revista eletrônica de biblioteconomia e ciência da informação, Ed Especial "Investigação em Sistemas de Informação", $2^{\mathrm{a}}$ Sem. Florianópolis, SC.

Khot, R.; Choppella, V.. (2011). DISCOVIR: A Framework for Designing Interfaces and Structuring Content for Virtual Labs. IEEE International Conference on Technology for Education. 


\section{CBIE-LACLO 2015}

Anais dos Workshops do IV Congresso Brasileiro de Informática na Educação (CBIE 2015)

Kitchenham, B. (2004). Procedures for Performing Systematic Reviews. Technical Report TR/SE-0401. Keele University. Acesso em: 08 jun. 2014.

Lorenzo, Carlos-Miguel; Sicilia, M.; Sánchez, S.. (2012). Studying the effectiveness of multi-user immersive environments for collaborative evaluation tasks. Revista Computers \& Education V. 59 E. 4, p. 1361-1376.

Mørch, A.; Hartley, M.; Ludlow, B.; Caruso, V; Thomassen, I. (2014). The Teacher as Designer: Preparations for Teaching in a Second Life Distance Education Course. IEEE 14th International Conference on Advanced Learning Technologies, At, Grécia.

Mota, F.; Espíndola, D.; Pinto, I.; Tusnski, L.. (2012). Um Caso de Uso do Ambiente Virtual de Aprendizagem (Sloodle) no Ensino à Distância. IX Congresso Brasileiro de Ensino Superior a Distância.

Nascimento, L.; Spilker, M.. (2012) Congresso Virtual no Second Life: Uma Atividade Imersiva na Perspetiva de Alunos do Mestrado em Pedagogia do E-Learning. II Congresso Internacional TIC e Educação.

Nunes, F. B.; Voss, G. B.; Herpich, F.; Mühlbeier, A.; Possobom, C. C.; Medina, R. D.. (2013). Viewers Para Ambientes Virtuais Imersivos: Uma Análise Comparativa Teórico-Prática. Revista Novas Tecnologias na Educação (RENOTE), V. 11 No 1.

Oliveira, L.; Espíndola, D.; Amaral, M.; Couto, Z.; Laurino, D.. (2014). O Design Participativo e as Ferramentas para a Autoria de Conteúdos em Ambientes Imersivos Educacionais. Revista Novas Tecnologias na Educação (RENOTE), V. 12 N $^{\circ} 2$.

Oliveira, L.; Espíndola, D.; Barwaldt, R.; Amaral, M.; Botelho, S. (2014b). Proposta de um Arcabouço Metodológico para a Autoria de Conteúdo em Ambientes Imersivos de Ensino. Revista Novas Tecnologias na Educação (RENOTE), V. 12 No 1.

Preece, J.; Rogers, Y.; Sharp, H.. (2005). Design de Interação: além da interação homem computador. Editora Bookman.

Schlemmer, E.; Backes, L.. (2008). Metaversos: novos espaços para construção do conhecimento. Rev Diálogo Educacional, Curitiba, V. 8, N² 24, p. 519-532.

Schlemmer, E.; Marson, F.. (2013). Immersive Learning: Metaversos e Jogos Digitais na Educação. 8th Iberian Conference on Information Systems and Technologies (CISTI), Lisboa, Portugal.

Tarouco, L.; Ávila, B.; Amaral, É.; Zednik, H.. (2012). VEGA - Implementando um Laboratório Virtual Imersivo no OpenSim. Revista Novas Tecnologias na Educação (RENOTE), V. $10 \mathrm{~N}^{\mathrm{o}} 1$.

Tarouco, L.; Gorziza, B.; Corrêa, Y.; Amaral, É.; Müller, T.. (2013). Virtual Laboratory for Teaching Calculus: an Immersive Experience. IEEE Global Engineering Education Conference (EDUCON), Berlim, Alemanha.

Viegas, S.C. (2012). Second Life: A New Approach In Professional Education In The Study Of Work Safety. IEEE Latin America Transactions, V. 10, No 1.

Wagner, R.; Moura, A.; Koslowski, S. R.; Passerino, L. M.; Piovesan, S. D.. (2012). VirtualTche - Mundo Imersivo do Instituto Federal Farroupilha - Campus Panambi. Revista Novas Tecnologias na Educação (RENOTE), V. 10 No 2. 